\title{
Fast and Global Minimization Convex Multiphase Active Contours Lifen Zhou ${ }^{1, a^{*}}$ and Shunji Zhang ${ }^{2, b}$ \\ 1, 2 School of Information Engineering, Qujing Normal University, Qujing 655011, Yunnan, China \\ a872053339@qq.com, b47903299 @qq.com
}

Keywords: Image segmentation; Active contour model; Multiphase; Global Minimization

\begin{abstract}
Biomedical images usually have multiple regions of interest, which lead to the study of multi object segmentation in this area. Multiphase level set active contour model using a gradient descent method to minimize energy non convex, so not only will be the local minimum, but also lead to erroneous segmentation, the fast algorithm can not be carried out, can not be solved quickly. Aiming at the above problems, we propose a multi object image segmentation algorithm of global convex coupling. First, in order to avoid the segmented regions overlap and vacuum, we put two-phase level set Chan Vese model was extended to four phase, and the introduction of edge stopping function improved regularization process, and then based on this model of convex optimization, to obtain the global optimal solution, finally, using dual minimization method for the fast calculation. Experimental results show that the proposed model can not only obtain the global optimal solution, but also greatly improve the efficiency and accuracy of the segmentation.
\end{abstract}

\section{Introduction}

Active contour model is very popular in image segmentation which is to obtain the input image region of interes, Chan-Vese[1] is proposed based on the Mumford Shah (M-S) model [2] no active edge contour model (C-V), it by two-phase symbol to distinguish between target and background of an image to be segmented, for biomedical image usually has multiple sense of interest in regional and regional characteristics of each different, so can't for complex image segmentation that is indivisible multiple images. CHUNG[3-4] etal proposed a multilevel level set method using a single level set to segment multiple objects. Each layer is provided with corresponding threshold value and the threshold value directly affects the segmentation result. VESE [5] proposed a multiphase level set model MLS, realize $2^{M}$ target segmentation with level set MLSM, Zheng Gang [6-7] combined with the background of filling technology and $\mathrm{C}-\mathrm{V}$ model, put forward the tree structure and pyramidal multiphase level set segmentation method of multi targets, the realization of $\mathrm{N}$ phase segmentation through $N-1$ level set segmentation can achieve convergence, multi sub target of weak edge. Weng Xiaoguang [8], the use of parallel regional segmentation, characteristics of serial high efficiency, proposes a segmentation method of $\mathrm{C}-\mathrm{V}$ model based on composite multiphase level set Wang Xue [9] proposed a multiphase level set collaborative spatial fuzzy clustering image segmentation algorithm for multi object, firstly using $\mathrm{C}$ - means for coarse segmentation of image fuzzy clustering algorithm, and then the initial function of compound operation as multiphase level set function. Active contour models from the literature [3-9] multiphase level set is the use of gradient method to realize the minimization of energy decreased, these methods are non convex, so the initialization sensitive, prone to local optimum problem.

Active contour model of energy minimization is commonly used method of convex optimization to solve the local minimum problem, and the convex optimization method is mainly for Heaviside function instead of the level set (or characteristic function) method can change the micro [0,1] in convex interval. Zach[10] and Lellman[11] proposed the convex minimization scheme of multiphase segmentation does not guarantee a global optimal solution of the original problem Moreno[12] proposed fast global convex contour model can guarantee the global optimum, but this model only uses the image region information, in the actual segmentation edge will appear the problem of inaccurate positioning. 
In this paper, we consider a globally convex version of the four phase piecewise constant energy functional motivated from the seminal work of Chan-Vese[1], By deriving an approximate novel convex functional we change the original formulation into a binary segmentation problem and utilize a Chambolle's dual minimization [14]to solve the relaxed formulation [15]. Combined with Split Bregman method, we propose a fast global convex contour model, compared with the traditional gradient descent method, this algorithm can find the global optimal solution, solve the edge segmentation positioning is not accurate, slow and other issues.

\section{Multiphase Active Contours Model}

Finding the Global Minimum. Chan-Vese[1] proposed model (C-V), Suppose that the image is composed of two homogeneous regions (object and background) composition, so the image can be approximated by two value image, namely by minimizing the energy functional to achieve the segmentation.

$$
E^{C V}(\phi)=\mu \int_{\Omega} \delta_{\varepsilon}(\phi)|\nabla(\phi)| d x+\lambda_{1} \int_{\Omega}\left|I-c_{1}\right|^{2} H_{\varepsilon}(\phi) d x+\lambda_{2} \int_{\Omega}\left|I-c_{2}\right|^{2}\left(1-H_{\varepsilon}(\phi)\right) d x
$$

where $\delta$ is the Dirac delta function, $c=\left(c_{1}, c_{2}\right)$ are the mean intensity values inside and outside regions respectively. The Euler-Lagrange equation with gradient descent formulation results in a nonlinear partial differential equation(PDE).

$$
\frac{\partial \phi}{\partial t}=\delta(\phi)\left(\operatorname{div}\left(\frac{\nabla \phi}{|\nabla \phi|}\right)-\lambda_{1}\left(I-c_{1}\right)^{2}+\lambda_{2}\left(I-c_{2}\right)^{2}\right)
$$

along with

$$
c_{1}=\frac{\int_{\Omega} I H(\phi) d x}{\int_{\Omega} H(\phi) d x} \quad c_{2}=\frac{\int_{\Omega} I(1-H(\phi)) d x}{\int_{\Omega}(1-H(\phi)) d x}
$$

$H(\phi)$ is the Heaviside function:

$$
H(\phi)=\left\{\begin{array}{l}
\text { lif } \varphi>0 \\
\text { Qif } \phi \leq 0
\end{array}\right.
$$

The level set method can easily solve the problem of curve evolution ,But the energy level sets is mainly affected by $H(\phi)$ and $H(\phi)$ is a binary function, not convex. Therefore, using level set method represents the energy functional is non convex, easy to fall into local optimum. In this paper, using the method of literature [16] non convex in the level set energy formula (2) is a convex optimization, a convex minimization problem.

$$
\min _{(c, \phi)} \varepsilon(c, \phi)=\int_{\Omega}|\nabla \phi| d x+\int_{\Omega}\left[\lambda_{1}\left(I-c_{1}\right)^{2}-\lambda_{2}\left(I-c_{2}\right)^{2}\right] \phi d x
$$

For any $\phi \in(-\infty,+\infty)$,In order to get a stable solution, reference[16-17] with $\phi$ value is defined in a convex set $[0,1]$ and $u$ instead of $\phi$ to show the difference with variables, a global minimizer can be found by carrying out the following minimization:

$$
\min _{0 \leq u \leq 1} \int_{\Omega}|\nabla u| d x+\int_{\Omega} \mid\left[\lambda_{1}\left(I-c_{1}\right)^{2}-\lambda_{2}\left(I-c_{2}\right)^{2}\right] u(x) d x
$$

Four Phase Model. Here we restrict our description to the four phase model which is used in segmenting images effectively. According to (5)and(6), allowing us to obtain the minimization: 


$$
\begin{aligned}
& \min _{0 \leq u_{1} \leq 1,0 \leq u_{2} \leq 1, c, \delta}\left\{F_{C}\left(u_{1}, u_{2}, c, \delta\right)\right. \\
& =\int_{\Omega}\left|\nabla u_{1}\right| d x+\int_{\Omega}\left|\nabla u_{2}\right| d x+\lambda_{11} \int_{\Omega}\left(I-c_{11}\right)^{2} u_{1} u_{2} d x \\
& +\lambda_{10} \int_{\Omega}\left(I-c_{10}\right)^{2} u_{1}\left(1-u_{2}\right) d x+\lambda_{01} \int_{\Omega}\left(I-c_{01}\right)^{2}\left(1-u_{1}\right) u_{2} d x \\
& \left.+\lambda_{00} \int_{\Omega}\left(I-c_{00}\right)^{2}\left(1-u_{1}\right)\left(1-u_{2}\right) d x\right\}
\end{aligned}
$$

along with

$$
\begin{gathered}
c_{11}=\frac{\int_{\Omega} I u_{1} u_{2} d x}{\int_{\Omega} u_{1} u_{2} d x} \quad c_{10}=\frac{\int_{\Omega} I u_{1}\left(1-u_{2}\right) d x}{\int_{\Omega} u_{1}\left(1-u_{2}\right) d x} \\
c_{01}=\frac{\int_{\Omega} I\left(1-u_{1}\right) u_{2} d x}{\int_{\Omega}\left(1-u_{1}\right) u_{2} d x} \quad c_{00}=\frac{\int_{\Omega} I\left(1-u_{1}\right)\left(1-u_{2}\right) d x}{\int_{\Omega}\left(1-u_{1}\right)\left(1-u_{2}\right) d x}
\end{gathered}
$$

In this paper, we propose a weighted modified regularization procedure to improve the energy minimization problem.

$$
\begin{aligned}
& \min _{0 \leq u_{1} \leq 1,0 \leq u_{2} \leq 1, c, \delta}\left\{F_{C}\left(u_{1}, u_{2}, c, \delta\right)\right. \\
= & \int_{\Omega} g\left|\nabla u_{1}\right| d x+\int_{\Omega} g\left|\nabla u_{2}\right| d x+\lambda_{11} \int_{\Omega}\left(I-c_{11}\right)^{2} u_{1} u_{2} d x \\
+ & \lambda_{10} \int_{\Omega}\left(I-c_{10}\right)^{2} u_{1}\left(1-u_{2}\right) d x+\lambda_{01} \int_{\Omega}\left(I-c_{01}\right)^{2}\left(1-u_{1}\right) u_{2} d x \\
& +\lambda_{00} \int_{\Omega}\left(I-c_{00}\right)^{2}\left(1-u_{1}\right)\left(1-u_{2}\right) d x
\end{aligned}
$$

along with

$$
g(\nabla I)=\frac{1}{1+\delta|\nabla I|^{2}}
$$

\section{Fast solution of Split Bregman}

The idea of split Bregman method is to convert the non constrained problem into an equivalent constraint problem, such as the coupling term and the nonlinear term, In this paper, using Split Bregman method for solving [18 -19 ] fast (9). The introduction of two vectors $d_{1}$ and $d_{2}$ were approximately $\nabla u_{1}$ and $\nabla u_{2}$ (9) can be rewritten as:

$$
\begin{aligned}
& \min _{0 \leq u_{1} \leq 1,0 \leq u_{2} \leq 1, c, \delta}\left\{F_{C}\left(u_{1}, u_{2}, c, \delta\right)\right. \\
& =\int_{\Omega} g\left|d_{1}\right| d x+\int_{\Omega} g\left|d_{2}\right| d x+\lambda \int_{\Omega} r_{1}(c) d x+\frac{\mu}{2} \int_{\Omega}\left|d_{1}-\nabla u_{1}\right|^{2} d x \\
& +\lambda \int_{\Omega} r_{2}(c) d x+\frac{\mu}{2} \int_{\Omega}\left|d_{2}-\nabla u_{2}\right|^{2} d x
\end{aligned}
$$

where the image region fitting terms are given by:

$$
r_{1}(c)=\left(\lambda_{11}\left(I-c_{11}\right)^{2}-\lambda_{10}\left(I-c_{10}\right)^{2}\right) u_{1}+\left(\lambda_{01}\left(I-c_{01}\right)^{2}-\lambda_{00}\left(I-c_{00}\right)^{2}\right)\left(1-u_{1}\right)
$$




$$
r_{2}(c)=\left(\lambda_{11}\left(I-c_{11}\right)^{2}-\lambda_{01}\left(I-c_{01}\right)^{2}\right) u_{2}+\left(\lambda_{10}\left(I-c_{10}\right)^{2}-\lambda_{00}\left(I-c_{00}\right)^{2}\right)\left(1-u_{2}\right)
$$

To ensure $d_{1}=\nabla u_{1}$ and $d_{2}=\nabla u_{2}$ was then introduced into the Bregman vector P1 and P2, with Bregman iteration type (11). The iterative optimization problem is:

$$
\left\{\begin{array}{l}
\left(u_{j}^{k+1}, d_{i}^{k+1}\right)=\arg \min _{u \in[0.1], d}\left\{\int_{\Omega} g\left|d_{i}\right| d x+\lambda \int_{\Omega} r_{j}(c) u_{j} d x+\frac{\mu}{2} \int_{\Omega}\left|d_{i}-\nabla u_{j}-p_{j}^{k}\right|^{2} d x\right\} \\
p_{j}^{k+1}=p_{j}^{k}+\nabla u_{j}^{k+1}-d_{i}^{k+1}
\end{array}\right.
$$

Solve for $u_{j}, d_{i}^{k+1}$ :

$$
\begin{gathered}
u_{j}=\arg \min _{u \in[0.1], d} \int_{\Omega} g\left|d_{i}\right| d x+\frac{\mu}{2} \int_{\Omega}\left|d_{i}-\nabla u_{j}-p_{j}^{k}\right|^{2} d x \\
d_{i}^{k+1}=\arg \min _{u \in[0.1], d} \lambda \int_{\Omega} r_{j}(c) u_{j} d x+\frac{\mu}{2} \int_{\Omega}\left|d_{i}-\nabla u_{j}-p_{j}^{k}\right|^{2} d x
\end{gathered}
$$

Where $k$ represents the first $k$ iteration, according to the variational principle, the optimal satisfies the Euler Lagrange equation followed by:

$$
\nabla u_{j}=\frac{\lambda}{\mu} r_{j}(c)+\nabla \bullet\left(d_{i}^{k}+p_{j}^{k}\right), u_{i} \in[0,1]
$$

where $\nabla \bullet$ Is the divergence operator, The available Gauss- Seidel iteration scheme for (12), the optimal $\nabla u_{j}$, Iterative discretization is expressed as:

$$
\left\{\begin{array}{l}
\alpha_{i, j}=\left(d_{i}\right)_{i-1, j}^{x, k}-\left(d_{i}\right)_{i, j}^{x, k}-\left(p_{j}\right)_{i-1, j}^{x, k}+\left(p_{j}\right)_{i, j}^{x, k}+\left(d_{i}\right)_{i, j-1}^{y, k}-\left(d_{i}\right)_{i, j}^{y, k}-\left(p_{j}\right)_{i, j-1}^{y, k}+\left(p_{j}\right)_{i, j}^{y, k} \\
\beta_{i, j}=\frac{1}{4}\left(\left(u_{j}\right)_{i-1, j}^{k}+\left(u_{j}\right)_{i, j-1}^{k}+\left(u_{j}\right)_{i+1, j}^{k}+\left(u_{j}\right)_{i, j+1}^{k}-\frac{\lambda}{\mu} r_{j}(c)+\alpha_{i, j}\right) \\
\left(u_{j}\right)_{i, j}^{k+1}=\max \left\{\min \left\{\beta_{i, j}, 1\right\}, 0\right\}
\end{array}\right.
$$

where $\alpha, \beta$ Is the intermediate variables, max and min are the maximum and minimum operators, the shrink operator is used to obtain the optimal solution $d_{i}^{k+1}$ :

$$
d_{i}^{k+1}=\max \left\{\left|\nabla u_{j}^{k+1}+p_{j}^{k}\right|-(\mu g)^{-1}, 0\right\} \frac{\nabla u_{j}^{k+1}+p_{j}^{k}}{\left|\nabla u_{j}^{k+1}+p_{j}^{k}\right|}
$$

Bregman iterative solution $u_{1}^{k+1}, u_{2}^{k+1}, d_{1}^{k+1}, d_{2}^{k+1}$.Compared with the (9) in the gradient descent method, all the algorithms are explicitly given expression, does not increase the complexity of the algorithm and when computing the $\nabla u_{j}$ used Gauss -Seidel iteration method, thus greatly improving the speed of the segmentation algorithm.

\section{Experimental Results and Analysis}

Simulation hardware environment: Lenovo laptop (pentium (R) Dual-Core CPU T4200 2.0GHz, 1.0GB RAM); Software Environment: Operating system is Windows 7, programming platform using Matlab7.6.

Figure 1 of a brain image $(257 * 197)$, a brain slice image segmentation $(119 * 78)$ control results, the two images are uneven gray images, From figure can see two algorithms can obtain desired segmentation results, however using gradient descent method converges to the CPU time for about 20 seconds, and the application of the proposed algorithm, convergence of the CPU time not to 1 
seconds, convergence speed greatly exceeds the gradient descent method. In fact, the proposed algorithm in all of the initial values can be automatically given, and using the gradient descent method often requires prior to artificially selection initial curve, reducing the degree of automation algorithm, and can be seen from the division, our model is better than higher accuracy $\mathrm{C}-\mathrm{V}$ model segmentation.
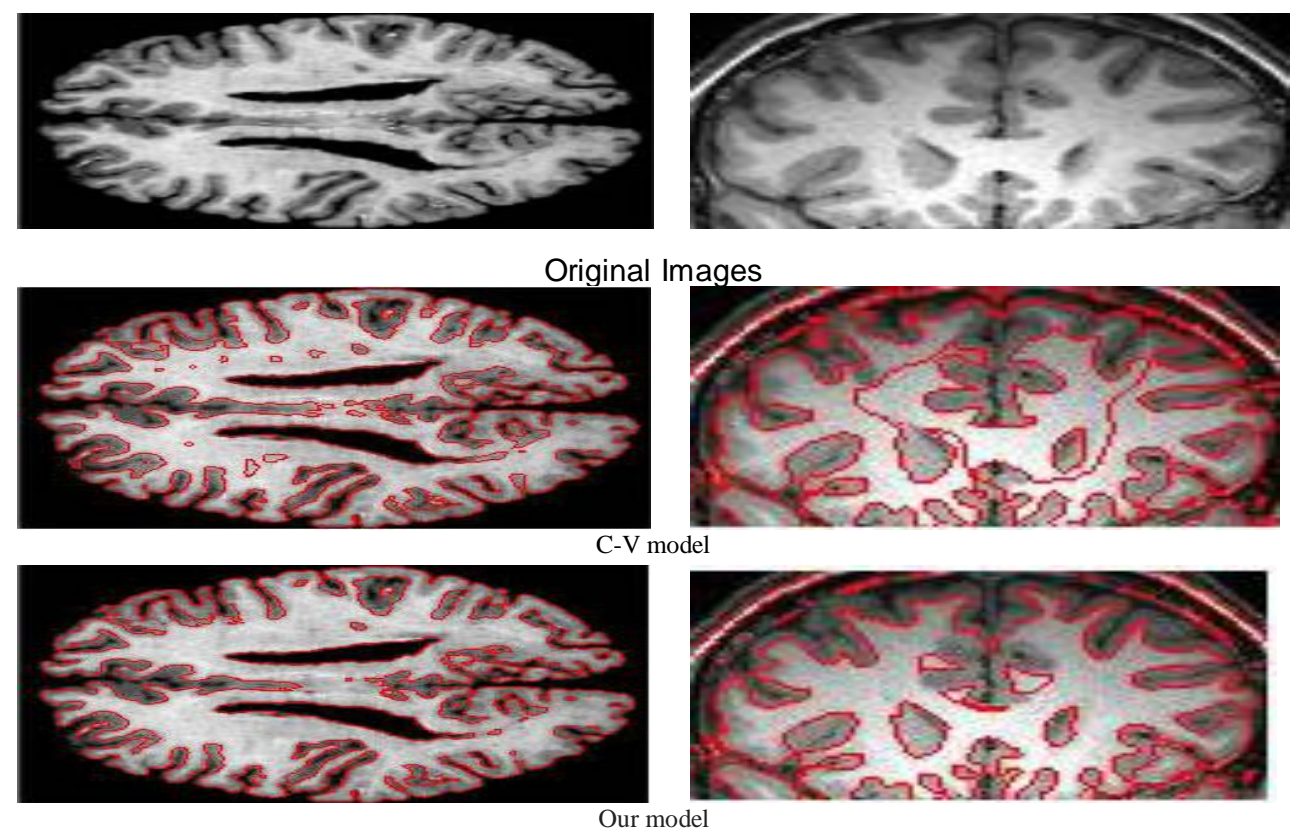

Figure 1. Comparison model

\section{Conclusions}

In this paper, we extend the four phases of two-phase $\mathrm{C}-\mathrm{V}$ model, model and convex optimization, so that the energy is convex to solve the segmentation result is easily trapped into local minima and some fast algorithms can not expand, in our proposed global convex contour model with edge information, making the edge location is separated more accurate, with the traditional gradient descent method compared, our proposed algorithm improves the segmentation accuracy, reduces the segmentation time.

\section{Acknowledgements}

We acknowledge partial funding by Qujing Normal University research project (2012QN024).

\section{References}

[1] T. F. Chan, S. Esedoglu, M. Nikolova, Algorithms for finding global minimizers of image segmentation and denoising models, SIAM Journal on Applied Mathematics 66 (2006) 1632-1648.

[2] T. F. Chan, L. A. Vese, Active contours without edges, IEEE Transactions on Image Processing 10 (2001) 266-277.

[3] CHUNG G , VESE LA. Energy minimization based segmentation and denoising using a multilayer level set approach[C]//Energy Minimization Methods in Computer Vision and Pattern Recognition, 2005: 439-455.

[4] CHUNG G , VESE LA. Image segmentation using a multilayer level-set approach[J]. Computing and Visualization in Science, 2009, 12(6): 267-285.

[5] VESE L A, CHAN T. A multiphase level set framework or image segmentation using the mumford and shah model[J]. International Journal of Computer Vision, 2002,50(3): 271-293. 
[6] ZHENG Gang, WANG Huinan, LI Yuanlu. A Tree LikeMultiphase Level Set A lgorithm for Image Segmentation Based on the Chan-V eseModel. Electronic journal, 2006, 34(8): 1508-1512.

[7] ZHENG Gang, WANG Huinan. A Pyramidal Multiphase Level Set Algorithm for Multi-object Segmentation of 3-D Medical Images. Journal Of Biomedical Engineering, 2008, 25(5): 989-993.

[8] WENG Xiao-guang, ZHENG Gang. Image Segmentation Based on Chan-Vese Model Using Composite Multiphase Level Set. Journal of Applied Sciences, 2009, 27(4): 365-369.

[9] WANG Xue LI Xuanping DAI Yixiang. Multiphase Level Set-based Multi-objective Image Segmentation Cooperating with Spatial Fuzzy C-means. Journal of Mechanical Engineering, 2013, 49(20): 10-15.

[10]Zach C, Gallup D, Frahm J M, et al. Fast Global Labeling for Real-Time Stereo Using Multiple Plane Sweeps[C]//VMV. 2008: 243-252.

[11]Lellmann J, Kappes J, Yuan J, etal.Convex multi-class image labeling by simplex-constrained total variation[M]//Scale Space and Variational Methods in Computer Vision. Springer Berlin Heidelberg, 2009: 150-162.

[12] Moreno J C, Surya Prasath V B, Proença H, et al. Fast and globally convex multiphase active contours for brain MRI segmentation. Computer Vision and Image Understanding, 2014.

[13]Pan Xiaohua, Sun Wenjie, Wei Zhihui. A Convex Model for Segmentation of Tissues for Brain MR Images Base on Mutual Information Maximizarion. Journal of Computer-Aided Design \& Computer Graphics, 2012, 24(8): 1082-1089.

[14] A. Chambolle, An algorithm for total variation minimization and applications,Journal of Mathematical Imaging and Vision 20 (2004) 89-97.

[15]J.-F. Aujol, G. Gilboa, T. Chan, S. Osher, Structure-texture image decomposition-modeling, algorithms and parameter selection, International Journal of Computer Vision 67 (2006) 111-136.

[16]Chan T F, Esedoglu S, Nikolova M. Algorithms for finding global minimizers of image segmentation and denoising models. SIAM journal on applied mathematics, 2006, 66(5): 1632-1648.

[17]Bresson X, Esedog lu S, Vandergheynst P, et al. Fast global minimization of the active contour/snake model[J]. Journal of Mathematical Imaging and vision, 2007, 28(2): 151-167.

[18] Goldstein T, Osher S. The split Bregman method for L1-regularized problems. SIAM journal on imaging sciences, 2009, 2(2): 323-343.

[19] Goldstein T, Bresson X, Osher S. Geometric applications of the split Bregman method: segmentation and surface reconstruction. Journal of Scientific Computing, 2010, 45(1-3): 272-293. 